\title{
Production of Individual Vaccine Component
}

National Cancer Institute

\section{Source}

National Cancer Institute. Production of Individual Vaccine Component. NCI Thesaurus.

Code C113050.

The preparation of material from one virus or bacteria strain for use as a component of a vaccine. 